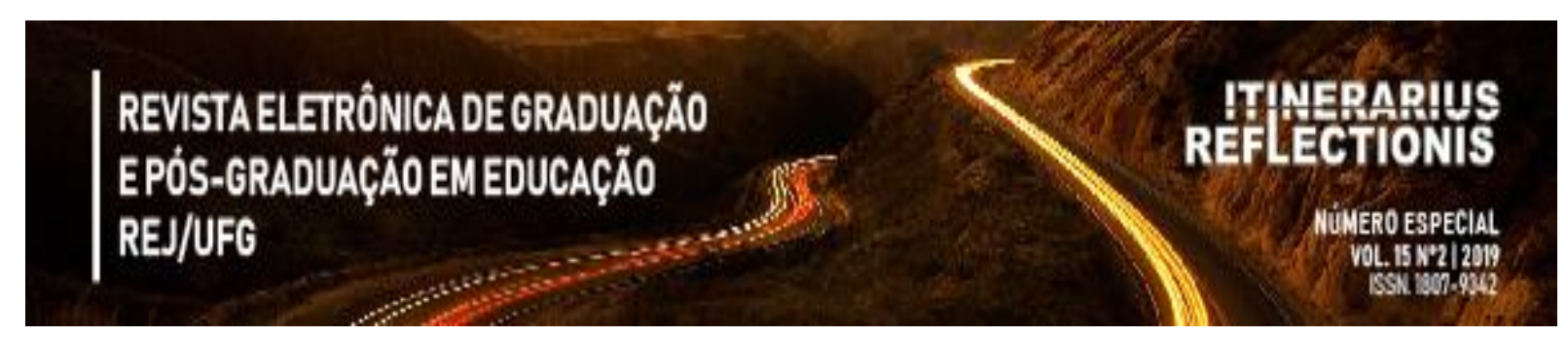

\title{
Identificando práticas pedagógicas no instagram: uma revisão sistemática
}

\author{
Priscila Campos Pereira ${ }^{1}$ \\ Flavio Ferreira Borges ${ }^{2}$ \\ Valquíria Perilo Sandoval Batista ${ }^{3}$ \\ Lucio França Teles ${ }^{4}$
}

Resumo. A tecnologia móvel tem proporcionado aos estudantes comodidade para acessar conteúdos que possam auxiliar em seu aprendizado. Nesse contexto, há indícios do uso da rede social Instagram em práticas pedagógicas. A rede social foi escolhida para esta análise devido ao crescimento significativo de usuários nos últimos anos, aumentando assim a sua presença na sala de aula. O objetivo desta pesquisa é mapear publicações relacionadas a práticas pedagógicas que utilizam o Instagram como ferramenta no apoio ao ensino. Os dados foram coletados entre os anos de 2014 a 2018 e disponível nas bases de dados científicas Web of Science, Scopus, DOAJ, ERIC, IRESIE e Redalyc. Os artigos foram analisados com o propósito de identificar quais são as ações práticas no processo ensino-aprendizagem com o uso do Instagram. A metodologia deste estudo foi a revisão sistemática a partir de uma lista inicial de 1.237 publicações. Após a verificação automática de repetições e ausência dos resumos, a lista final para leitura dos resumos foi de 947 artigos. A definição da quantidade de artigos a sem lidos de forma completa foi estabelecida a partir da identificação de práticas pedagógicas utilizando o aplicativo, sendo selecionadas 20 publicações a partir da atribuição de uma nota entre 1 e 5 . Foi possível identificar mudanças nas práticas dos professores com o propósito de aproximar dos saberes tecnológicos dos alunos, e a partir deste entendimento, promover uma aprendizagem de forma participativa e colaborativa. Todavia ainda não ocorre de forma efetiva.

Palavras-chave: Educação. Internet. Rede Social. Instagram.

\section{Identifying pedagical pratices in instagram A systematic review}

\begin{abstract}
Mobile technology has provided students with convenience to access content that can aid in their learning. In this context, there are indications of the use of the Instagram social network in pedagogical practices. The social network was chosen for this analysis due to the significant growth of users in recent years, thus increasing its presence in the classroom. The objective of this research is to map publications related to pedagogical practices that use Instagram as a tool to support teaching. Data were collected between the years 2014 and 2018 and are available in the scientific databases Web of Science, Scopus, DOAJ, ERIC, IRESIE and Redalyc. The articles were analyzed with the objective of identifying practical actions in the teaching-learning process with the use of Instagram. The methodology of this study was the systematic review from an initial list of 1,237 publications. After the automatic verification of repetitions and absence of abstracts, the final smooth reading of abstracts was

\footnotetext{
${ }^{1}$ Mestranda em Educação, Faculdade de Educação, Universidade de Brasília, priscila.pcp@ gmail.com.

${ }^{2}$ Doutorando em Educação, Faculdade de Educação, Universidade de Brasília, ferreiraflavioborges@gmail.com

${ }^{3}$ Mestranda em Educação, Faculdade de Educação, Universidade de Brasília, valquiriaperilo@gmail.com

${ }^{4}$ Faculdade de Educação, Universidade de Brasília, teleslucio@ gmail.com
} 
947 articles. The definition of the number of articles to be read in a complete way was established from the identification of pedagogical practices using the application, being selected 20 publications from the attribution of a grade between 1 and 5. It was possible to identify changes in the practices of teachers with the purpose of approaching the students' technological knowledge, and from this understanding, to promote a participatory and collaborative learning. However, it has not yet occurred effectively.

Keywords: Education. Internet. Social Network. Instagram.

\section{INTRODUÇÃO}

Segundo Castells (1999), a internet é o centro contemporâneo do padrão sociotécnico que repercute na sociedade atual formando a base material das relações humanas, tanto no trabalho como na comunicação. Nesse viés, propicia o processamento da virtualidade e da propagação de ideias entre os indivíduos, constituindo assim a sociedade em rede. A sociabilidade está em constante transformação em âmbito de redes pessoais, principalmente na internet, onde as redes sociais disseminadas em comunidades virtuais possibilitam que as pessoas troquem informações e promovam assim uma aprendizagem em um espaço informal.

A sociedade contemporânea tem observado crescente trocas de informações com pessoas do mundo inteiro. Lévy (1996) afirma que os grupos de pessoas estão sendo formados independentemente da localização geográfica, por meio das comunidades virtuais. Nesse contexto, destaca-se a tecnologia móvel, que permite acessar variadas informações, comunicarse em diferentes formatos, realizar transações bancárias e aprender os mais diversos assuntos.

A busca por um ensino de qualidade sempre esteve permeando os anseios da sociedade (MORAN, 2006). Há uma inquietação para que os professores sejam inovadores em suas práticas pedagógicas, com o intuito de aproximar dos saberes tecnológicos desses alunos originados de uma sociedade digital (FERREIRA-BORGES; FRANÇA-TELES, 2019). O professor que permitir o novo, o desconhecido em seu universo de atuação descobrirá como viabilizar as tecnologias nas práticas pedagógicas. Entre essas possibilidades ressaltam-se as redes sociais como meio de fomentar o processo educativo.

Atualmente existem várias redes sociais. O Instagram encontra-se na sexta posição em número de seguidores, com 813 milhões de contas em abril de 2018 (STATISTA, 2018), sendo utilizado para compartilhar fotos, vídeos e curtas mensagens textuais. Dessa forma, 


\section{REVISTAELETRÔNICADEGRADUACÄO}

EPÓS-GRADUAÇÄO EM EDUCAÇÄO REJ/UFG

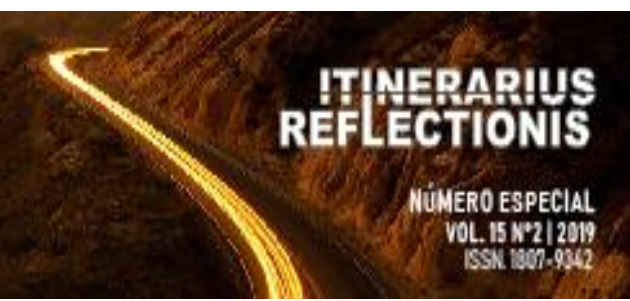

utilizá-lo no contexto educativo se torna pertinente, dado que o seu crescimento nos últimos quatro anos foi na ordem de $450 \%$, conforme gráfico de crescimento apresentado na figura 1 .

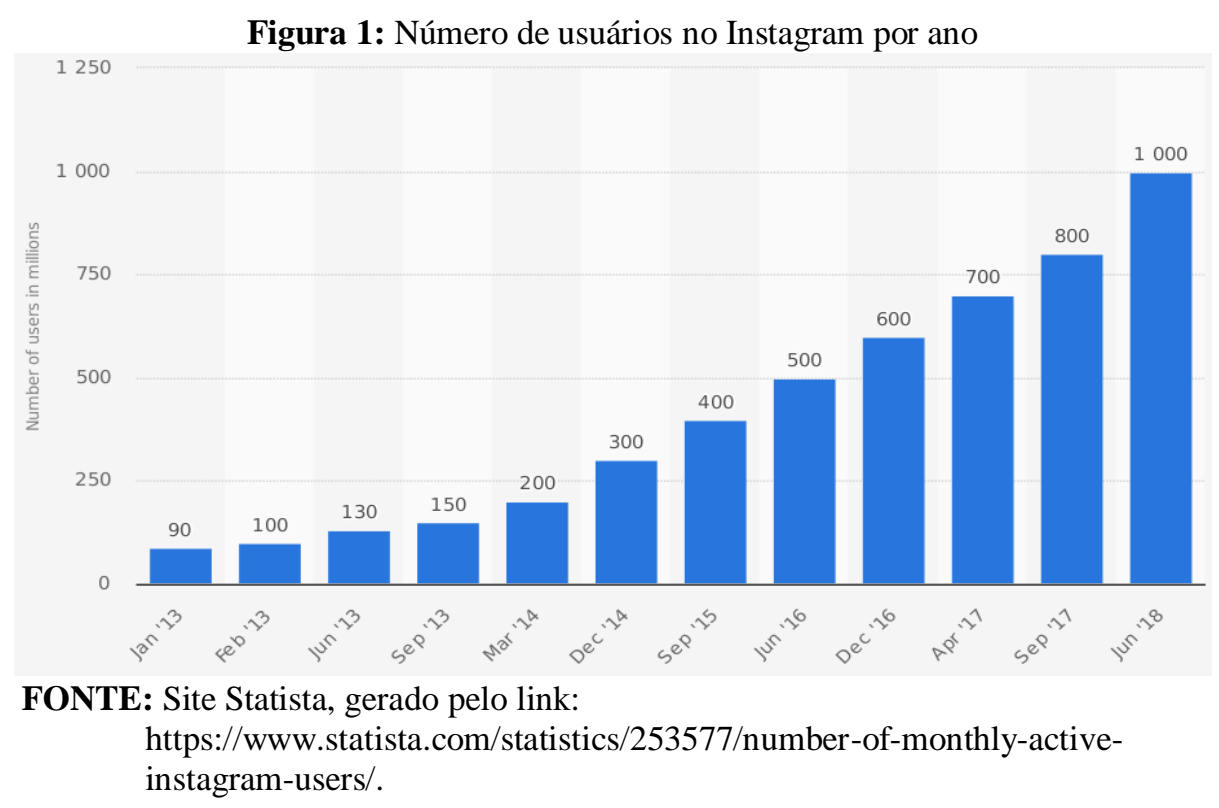

Para averiguar se o crescimento na utilização do Instagram provocou alguma mudança nas práticas pedagógicas de professores que usam as tecnologias em sala de aula, foi realizada uma revisão sistemática (RS) para identificar artigos científicos que apresentassem informações sobre o uso dessa rede social em sala de aula ou fora dela com o propósito de efetivar uma aprendizagem significativa dos estudantes.

Uma RS apoiada por uma revisão da literatura torna-se uma metodologia precisa, rigorosa e transparente para identificar um modelo ou estudo (COFFIELD et al., 2004) e deverá ser planejada com critérios que possam ser verificados e reutilizados por outros pesquisadores, quando da necessidade de comprovarem os resultados de uma pesquisa (DE-LA-TORREUGARTE-GUANILO; TAKAHASHI; BERTOLOZZI, 2011). Esse estudo revisou e sintetizou sistematicamente a literatura relevante sobre a utilização de rede social em práticas pedagógicas, e os critérios estão devidamente explicados na seção sobre a metodologia.

\section{O USO DAS TECNOLOGIAS DIGITAIS: REFLEXÕES NO PROCESSO DE ENSINO E APRENDIZAGEM}




\section{REVISTAELETRÔNICADEGRADUACÄOO}

EPÓS-GRADUAÇÄO EM EDUCAÇÄO

REJ/UFG

O transcurso civilizatório do homem é marcado pelos diferentes formatos de comunicação para a realização do processo educativo. Inicialmente, apresentou-se a forma de ensinar com prevalência da presença física entre as pessoas, apesar de pouco diálogo entre elas. Predominava a forte centralidade no professor, que dispunha do conhecimento, e cabia aos estudantes se submeterem a ele com passividade extrema. Dessa forma, Kenski (2008a, p. 10) apresenta como exemplo a maiêutica, em que "o mestre encarnava o conhecimento a ser aprendido, ele tinha também todo o domínio sobre o que deveria ser ensinado". Segundo a autora (KENSKI, 2008b), a tecnologia caracteriza-se por ser um complexo de saberes e fundamentos científicos que podem ser propiciados na construção, no planejamento e na utilização de um suporte para o andamento de uma atividade específica.

Pierre Lévy (1996) preconiza, com uma certa preocupação, a importância da tecnodemocracia na vida das pessoas, pois ela possibilita uma apropriação das tecnologias para um bem comum, criando redes e relacionamentos entre tudo e todos, promovendo o surgimento das tecnologias da inteligência, ou seja, o conhecimento.

Nesse contexto, os tipos de tecnologia podem ser relacionados à linguagem oral, à escrita e à digital. Ademais, urge destacar as Tecnologias de Informação e Comunicação (TIC), que permitem transformações significativas no contexto social nas áreas da comunicação e da educação. Com essas tecnologias, é possível acessar as mais variadas informações em contexto global, bem como transmitir em articulação comunicativa.

$\mathrm{O}$ uso das tecnologias no contexto educacional ocorre de diferentes maneiras. Destaca-se que não é somente o uso das tecnologias como fim de si mesmas que torna o aprendizado dos estudantes significativo. É importante compreender o papel do professor na modernização dos processos de ensino-aprendizagem, a pesquisa de McKnight et al. (2016) confirma a necessidade de um conhecimento prévio dessas tecnologias antes de usá-las nos sistemas tradicionais de ensino, com o objetivo de transformá-las em ambientes digitais.

Kenski (2008b, p. 9) argumenta que "não sabe dizer até que ponto vamos falar de novos processos de interação e de comunicação, ou se falamos dos mesmos processos, a partir de uma nova ótica". De fato, quando trabalhamos a mediação nas Tecnologias de Comunicação e Informação (TIC), é necessário buscar agregar todos os envolvidos a fim de que tenham 


\section{REVISTAELETRÔNICADEGRADUACÄO}

EPÓS-GRADUAÇÄO EM EDUCAÇÄO REJ/UFG

REFITECTIONIS

NUMERO ESPECIAL

VoL. $15 K^{\circ} 212019$

[SSN TOM. 942 ?

realmente a interação e o processo comunicativo. Nesse sentido, a aprendizagem móvel atrelada às TIC facilita o acesso dos indivíduos aos mais variados conhecimentos de forma interativa.

A Unesco (2014) define tecnologia móvel em caráter mais amplo, a saber: "aparelhos digitais, facilmente portáteis, de propriedade e controle de um indivíduo, com capacidade de acesso à internet e aspectos multimídia, e que podem facilitar muitas tarefas, particularmente aquelas relacionadas à comunicação".

As redes sociais, por conseguinte, são espaços cada vez mais utilizados pela sociedade contemporânea. Uma das características dessas redes é favorecer espaços para compartilhar fotos, notícias e opiniões. Os variados aspectos multimídia dentro das redes sociais oportuniza a aprendizagem significativa. Assim, utilizá-las no contexto escolar possibilita ampliar os espaços educativos.

Santos e Santos (2015) explana que o momento histórico técnico-científicoinformacional, por meio das TIC - como computadores, celulares, smartphones, tablets -, “enquanto possibilidade de comunicação e informação, está modificando a maneira como as pessoas se relacionam, aprendem e se comunicam". Nesse contexto, o aplicativo Instagram desenvolvido por Kevin Systrom e pelo brasileiro Mike Krieger em 2010 - proporciona o contato direto entre várias pessoas que estão utilizando recursos de seguir alguém, curtir, comentar e compartilhar conteúdos por meio de hashtags (\#).

Em suma, o Instagram no contexto educativo possibilita otimizar ações pedagógicas, por meio de textos curtos, imagens e vídeos. Dessa forma, a investigação dos estudos recentes dessa rede social no contexto educativo a partir da RS buscou ratificar as repercussões em foco

\section{PRÁTICAS EDUCATIVAS NA REDE SOCIAL INSTAGRAM: UMA REVISÃO SISTEMÁTICA}

A RS inicia-se pela formulação de uma pergunta clara e objetiva sobre determinado assunto do qual se pretende o maior esclarecimento. Deve-se utilizar métodos sistemáticos e explícitos que promovam condições de analisar as informações contidas nas publicações selecionadas (GALVÃO; PANSANI; HARRAD, 2015). Foram levadas em consideração as 


\section{REVISTAELETRÔNICADEGRADUACÄO}

EPÓS-GRADUAÇÄO EM EDUCAÇÄO REJ/UFG

orientações do Prisma ${ }^{5}$, protocolo que propõe um checklist de 27 itens que devem constar na elaboração de uma RS, como: descrever as fontes de informação corretamente; apresentar a estratégia de busca e seleção dos artigos; informar como se deu o processo de coleta de dados; expor variáveis obtidas e outras.

A primeira etapa de uma RS é definir quais questões podem ser elaboradas para a construção dos termos de buscas e, desses termos, construir o critério da busca a ser realizada (SONG, 2014). Para nortear este objetivo, foram elaboradas duas questões:

- O artigo faz referência direta ao uso do Instagram em atividades pedagógicas?

- O artigo sugere ou apresenta alguma atividade prática para o uso do Instagram com propósito educacional?

Para responder a essas perguntas, foram definidas como critério de busca três palavras-chave: Instagram, educação e ensino. A construção do termo utilizado para fazer a pesquisa nas bases de dados foi permeada pelo conectivo $A N D$, a fim de obter o combinado das palavras em artigos científicos. Salienta-se que todas as palavras estavam em maiúsculo para a maior precisão de dados. Sendo assim, os termos tiveram como propósito fornecer aos mecanismos de busca uma orientação sobre quais artigos deveriam constar na lista de resultado das pesquisas, conforme descrição apresentada no quadro 1. Não houve restrição sobre em qual seção ou em que sequência os termos aparecem no texto, contudo, os dois termos deveriam estar presentes, sem exceção.

Quadro 1: construção dos termos de busca com o conectivo AND.

\begin{tabular}{|l|l|}
\hline Instagram AND Educação & Instagram AND Education \\
\hline Instagram AND Ensino & Instagram AND Teaching \\
\hline $\begin{array}{l}\text { A busca deverá retornar somente artigos que conste as duas palavras } \\
\text { informadas, em qualquer seção do documento. }\end{array}$
\end{tabular}

FONTE: elaborado pelos autores/autoras.

\footnotetext{
${ }^{5}$ http://www.prisma-statement.org/; (GALVÃO; PANSANI; HARRAD, 2015)
} 


\section{REVISTAELETRÔNICADEGRADUAÇ̈̈O}

EPÓS-GRADUAÇ̄OOEM EDUCAÇÄO REJ/UFG

\section{ITINERARUS REFLECTIONIS}

NÜERO ESPECIAL

VOL. $15 K^{+} 212019$

[SSN

As bases de dados escolhidas para a realização da pesquisa foram DOAJ, ERIC,

IRESIE, Redalyc, Scopus e Web Of Science (Scielo BR). Essas bases foram consideradas em virtude dos critérios de classificação Qualis/Capes (CAPES, 2014) por indexar diversas revistas científicas e são consideradas no ranqueamento da instituição, conforme a sua área de conhecimento, podendo chegar até o nível A1.

As consultas foram realizadas nas seis bases e utilizaram os termos apresentados no quadro 1 - sem qualquer filtro de restrição, como ano de publicação -, revisados por pares ou idioma. A exceção foi a base de dados Redalyc, na qual foi necessário marcar o filtro de publicações somente da área educação, em virtude de o mecanismo de busca da base não aplicar corretamente o uso do conectivo AND.

O trabalho nas bases foi feito por um pesquisador e duas pesquisadoras e, na primeira interação da pesquisa, quando os resultados foram unificados em uma planilha, foram identificados 1.237 artigos científicos. A lista final foi compilada, retirando as publicações repetidas e sem resumo, resultando em 947 artigos. Foram acrescentados 14 artigos sugeridos por estudantes e especialistas em educação participantes da disciplina Aprendizagem, Tecnologia e Educação a Distância, ministrada pelo professor Dr. Lucio França Teles no programa de Pós-Graduação em Educação da Faculdade de Educação da UnB, na qual um ensaio desse trabalho foi apresentado. Urge destacar que essas publicações estão identificadas como "base não informada". Por fim, as quantidades por base estão sendo representadas na figura 2 para maior esclarecimento.

Figura 2: quantidade de publicações selecionadas por base 


\section{REVISTAELETRÔNICADEGRADUACÄO}

EPÓS-GRADUAÇÄO EM EDUCAÇÄO REJ/UFG
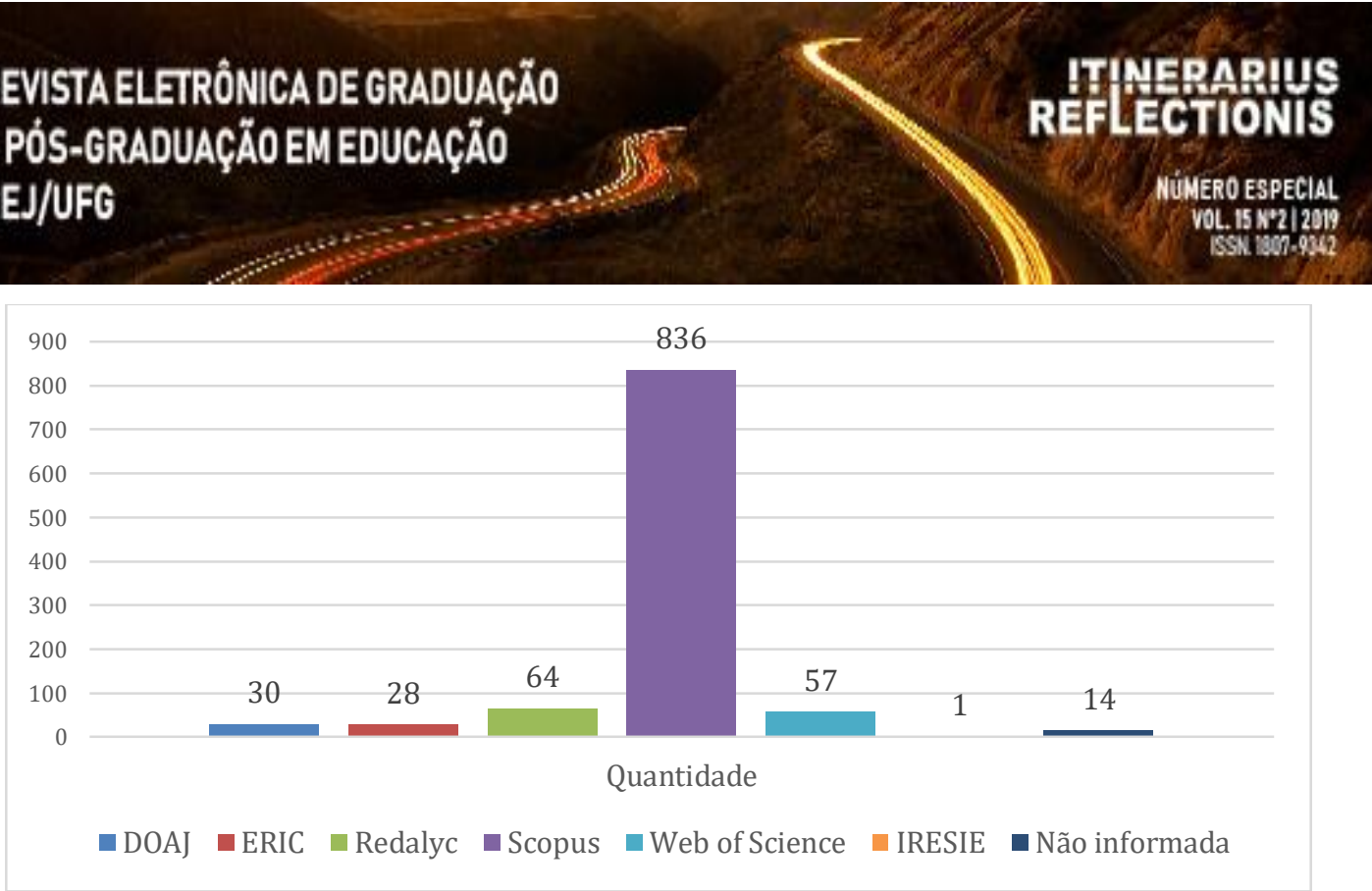

FONTE: elaborado pelos autores/autoras.

Após a seleção dos artigos, iniciou-se o processo de avaliação para verificar se a abordagem do trabalho possibilitava responder às duas questões norteadoras desta pesquisa. Desse modo, os títulos e resumos dos 947 artigos foram lidos por três pesquisadores. Adiante, cada avaliador atribuiu uma nota em uma escala de 1 a 5 , em que 1 indicava que a proposta não abordava a temática em nenhum requisito e 5 indicava que a proposta era relevante e possibilitaria responder aos dois questionamentos propostos.

Foram selecionados para análise os 20 artigos que obtiveram nota mínima 3 atribuída pelos três avaliadores, ou seja, se um artigo recebesse uma nota menor que 3 por um dos avaliadores, era retirado da lista. O quadro 2 apresenta a lista dos artigos selecionados, e a figura 3 mostra o gráfico sobre a quantidade de artigos avaliados conforme a nota atribuída pelo avaliador.

Quadro 2: Os 20 artigos selecionados após atribuição da pontuação com a indicação dos termos utilizados para recuperá-los e a base de dados de origem.

\begin{tabular}{|c|l|l|}
\hline Termos & Base & Título \\
\hline \multirow{5}{*}{ Instagram } & Redalyc & $\begin{array}{l}\text { Formação e ferramentas colaborativas para a docência na web social. } \\
\text { (MOREIRA; MONTEIRO, 2015) }\end{array}$ \\
\cline { 3 - 3 } & $\begin{array}{l}\text { Social media in undergraduate learning: categories and characteristics. } \\
\text { (SMITH, 2017) }\end{array}$ \\
\cline { 2 - 3 } & $\begin{array}{l}\text { University students' self-regulated learning using digital technologies. } \\
\text { (YOT-DOMÍNGUEZ; MARCELO, 2017) }\end{array}$ \\
\hline
\end{tabular}




\section{REVISTAELETRÔNICA DE GRADUAÇÄO EPÓS-GRADUAÇÄO EM EDUCAÇÄO REJ/UFG}

\begin{tabular}{|c|c|c|}
\hline \multirow{5}{*}{$\begin{array}{l}\text { Instagram AND } \\
\text { education }\end{array}$} & \multirow{2}{*}{ DOAJ } & $\begin{array}{l}\text { Incorporating Memes and Instagram to Enhance Student's Participation. } \\
\text { (PURNAMA, 2017) }\end{array}$ \\
\hline & & $\begin{array}{l}\text { Integration of Education: Using Social Media Networks to Engage } \\
\text { Students. (BLAIR; SERAFINI, 2014) }\end{array}$ \\
\hline & Scopus & Private open source social networking media for education. (NINI, 2015) \\
\hline & \multirow[t]{2}{*}{$\begin{array}{l}\text { Web of } \\
\text { Science }\end{array}$} & $\begin{array}{l}\text { Application of Social Media in Chemistry Education: Incorporating } \\
\text { Instagram and Snapchat in Laboratory Teaching. (LIM; ANG; FUNG, } \\
\text { 2017) }\end{array}$ \\
\hline & & Gamification in education: social network analysis. (DICHEVA, 2017) \\
\hline \multirow{4}{*}{$\begin{array}{l}\text { Instagram AND } \\
\quad \text { learning }\end{array}$} & & $\begin{array}{l}\text { Instagram: una experiència de telecol-laboració australianocatalana. } \\
\text { (PEDRÓ-GARCÍA, 2016) }\end{array}$ \\
\hline & DOAJ & $\begin{array}{l}\text { Pengembangan Media Pembelajaran Berupa Komik Fisika Berbantuan } \\
\text { Sosial Media Instagram sebagai Alternatif Pembelajaran. (IRWANDANI; } \\
\text { JUARIYAH, 2016) }\end{array}$ \\
\hline & Scopus & $\begin{array}{l}\text { Where you Instagram? Associating your Instagram photos with points of } \\
\text { interest. (XUTAO et al., 2015) }\end{array}$ \\
\hline & $\begin{array}{l}\text { Web of } \\
\text { Science }\end{array}$ & $\begin{array}{l}\text { Utilização do Instagram no ensino e aprendizagem de português língua } \\
\text { estrangeira por alunos Chinese da University of Aveiro. (BARBOSA et } \\
\text { al., 2017) }\end{array}$ \\
\hline \multirow{8}{*}{$\begin{array}{l}\text { Instagram AND } \\
\text { teaching }\end{array}$} & \multirow{3}{*}{ ERIC } & $\begin{array}{l}\text { Concept Mapping: An "Instagram" of Students' Thinking. (CAMPBELL, } \\
\text { 2016) }\end{array}$ \\
\hline & & $\begin{array}{l}\text { Incorporating Twitter, Instagram, and Facebook in Economics } \\
\text { Classrooms. (AL-BAHRANI; PATEL, 2015) }\end{array}$ \\
\hline & & $\begin{array}{l}\text { Using WhatsApp to Enhance Students' Learning of English Language } \\
\text { "Experience to Share". (HAMAD, 2017) }\end{array}$ \\
\hline & \multirow{4}{*}{ Scopus } & $\begin{array}{l}\text { Engaging students using social media: The students' perspective. (AL- } \\
\text { BAHRANI; PATEL; SHERIDAN, 2015) }\end{array}$ \\
\hline & & $\begin{array}{l}\text { Harnessing a Mobile Social Media App to Reinforce Course Content. } \\
\text { (KORICH, 2016) }\end{array}$ \\
\hline & & $\begin{array}{l}\text { Learning through social media: Students' perception. (JAMARI et al., } \\
\text { 2014) }\end{array}$ \\
\hline & & $\begin{array}{l}\text { Students' perceptions and attitudes towards the use of Instagram in English } \\
\text { language writing. (AKHIAR et al., 2017) }\end{array}$ \\
\hline & $\begin{array}{l}\text { Web of } \\
\text { Science }\end{array}$ & $\begin{array}{l}\text { Web social y alfabetización informacional: experiencia en la Universidad } \\
\text { de Puerto Rico. (PAGÁN; ORTIZ, 2017) }\end{array}$ \\
\hline
\end{tabular}


REVISTAELETRÔNICADE GRADUAÇÄO

EPÓS-GRADUAÇÄO EM EDUCAÇÄO

REJ/UFG

FONTE: elaborado pelos autores/autoras.

Figura 3: Quantidade de notas atribuídas por avaliador conforme o grau de relevância do trabalho para a revisão sistemática proposta.

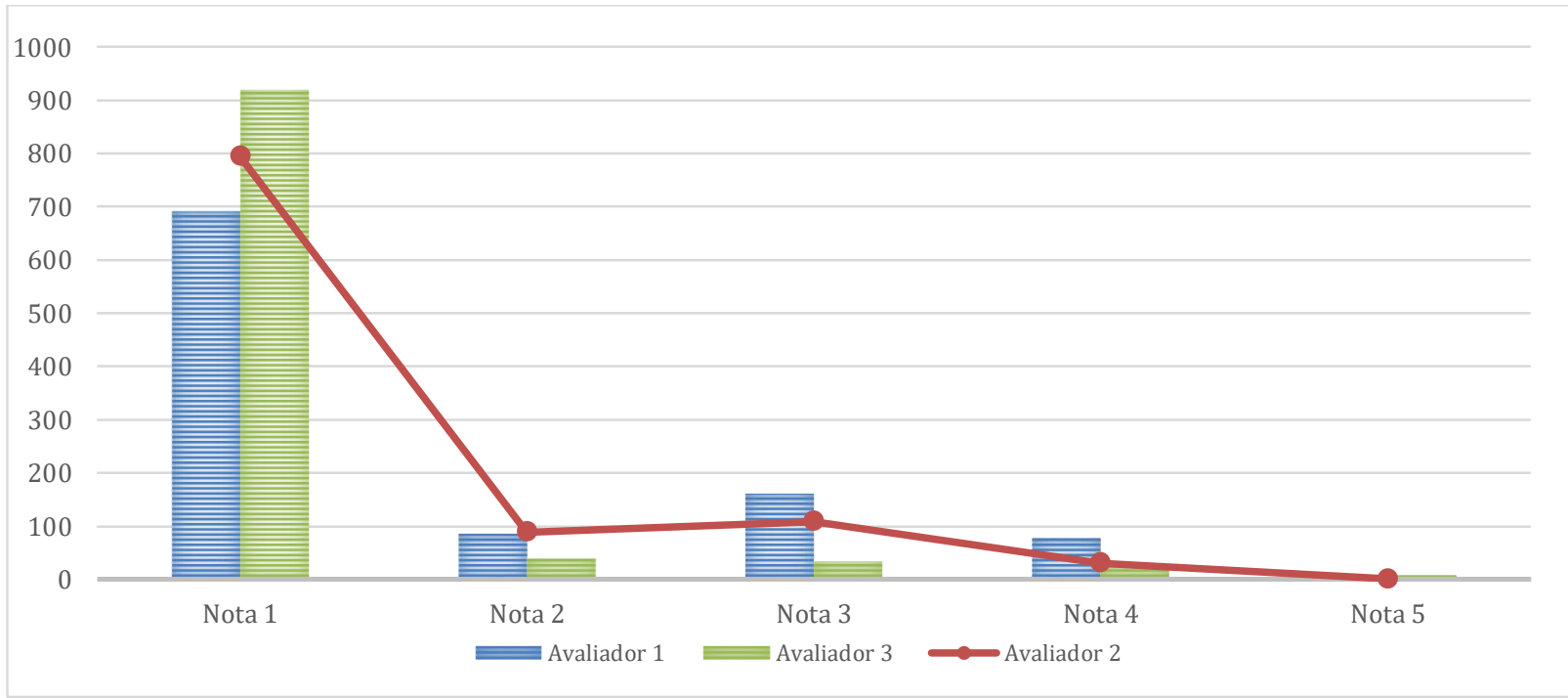

FONTE: elaborado pelos autores/autoras.

Com o propósito de averiguar se as publicações selecionadas estão alinhadas com pesquisas na área da educação, redes sociais e Instagram, foi criada uma imagem (figura 4) para visualizar as palavras que mais apareceram nos 20 artigos. Este recurso visual é conhecido como nuvem de palavras.

Figura 4: Nuvem de Palavras dos textos presentes nos 20 artigos selecionados. 

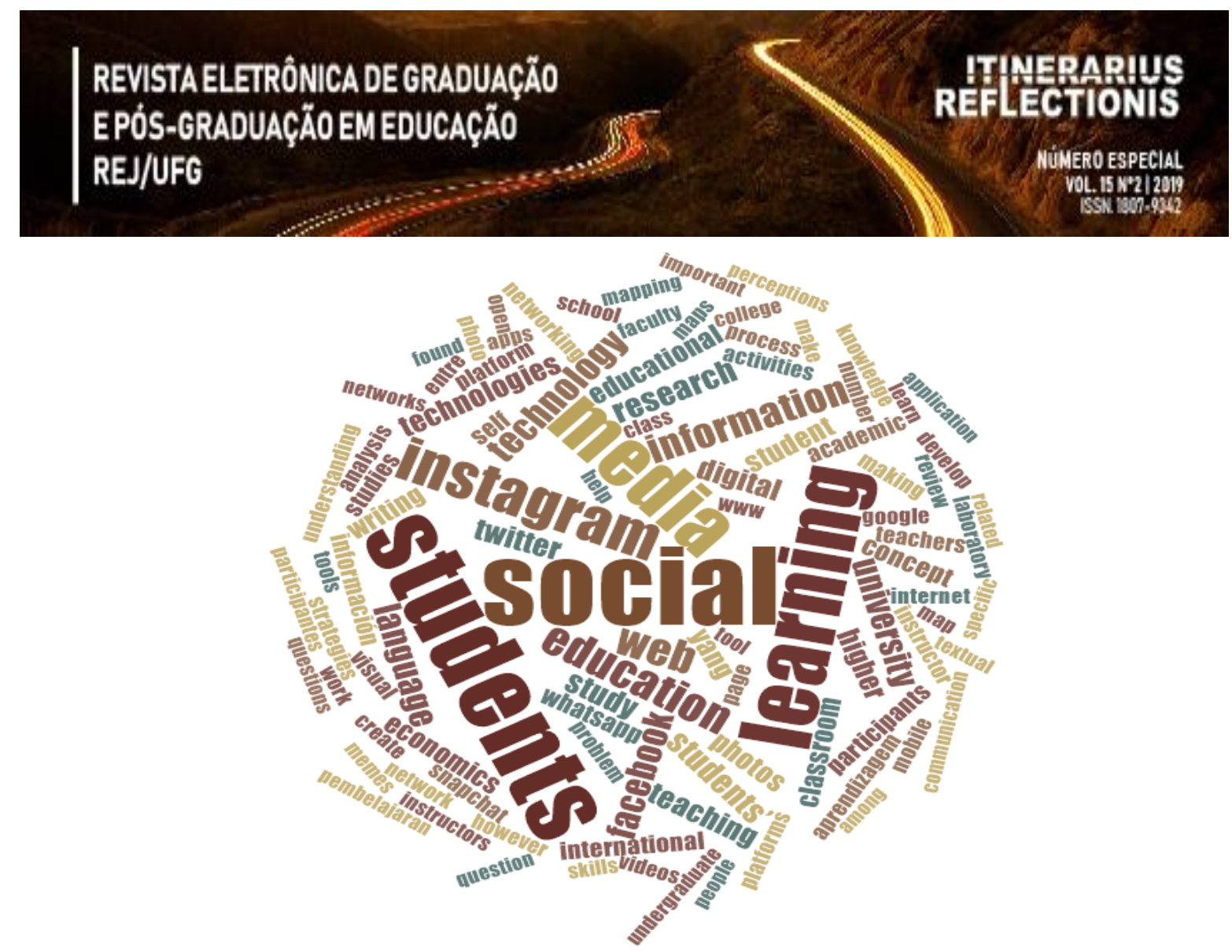

FONTE: elaborado pelos autores/autoras.

O propósito da nuvem de palavras apresentado na figura 4 é identificar os termos que aparecem com mais frequência em um texto ou em vários arquivos de texto. Como podemos observar, as palavras "students", "learning", "media", "social", "Instagram", "web" e “education" são as que mais se destacaram nas publicações - em virtude do tamanho da fonte -, demonstrando que os artigos selecionados abordam a temática dessa pesquisa. Na próxima seção, será produzida uma análise sintetizada das propostas identificadas.

\section{SÍNTESE E REFLEXÕES SOBRE AS PROPOSTAS}

As propostas selecionadas concentram suas pesquisas em práticas do ensino superior, sendo $75 \%$ em ambientes universitários, $15 \%$ em todos os níveis e 10\% em propostas direcionadas ao ensino fundamental. Sobre o público alvo, considerando o principal objeto de estudo, os alunos representam $60 \%$ desse foco e os professores $20 \%$, e os demais estudos estão divididos entre propostas institucionais, sugestões de mais pesquisas e ao setor de educação em geral.

Ao classificar as propostas por área de conhecimento, tendo como referência a educação como principal objeto de estudo, o ensino de língua estrangeira, economia e química 


\section{REVISTAELETRÔNICADEGRADUAÇÄO}

EPÓS-GRADUAÇÄO EM EDUCAÇÄO REJ/UFG

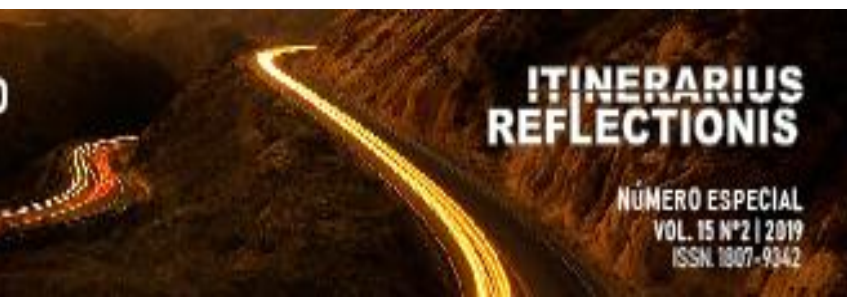

foram as disciplinas que mais se destacaram entre as pesquisas. Os pesquisadores, em sua grande maioria professores de centro universitário, buscam responder seus questionamentos analisando e propondo ações práticas no uso das redes sociais de uma forma geral, seja com imagens, textos ou a combinação dos dois. A figura 5 retrata a distribuição desses pesquisadores conforme os países vinculados nas instituições informadas nos dados dos autores.

Figura 5: distribuição das publicações conforme o País dos autores.

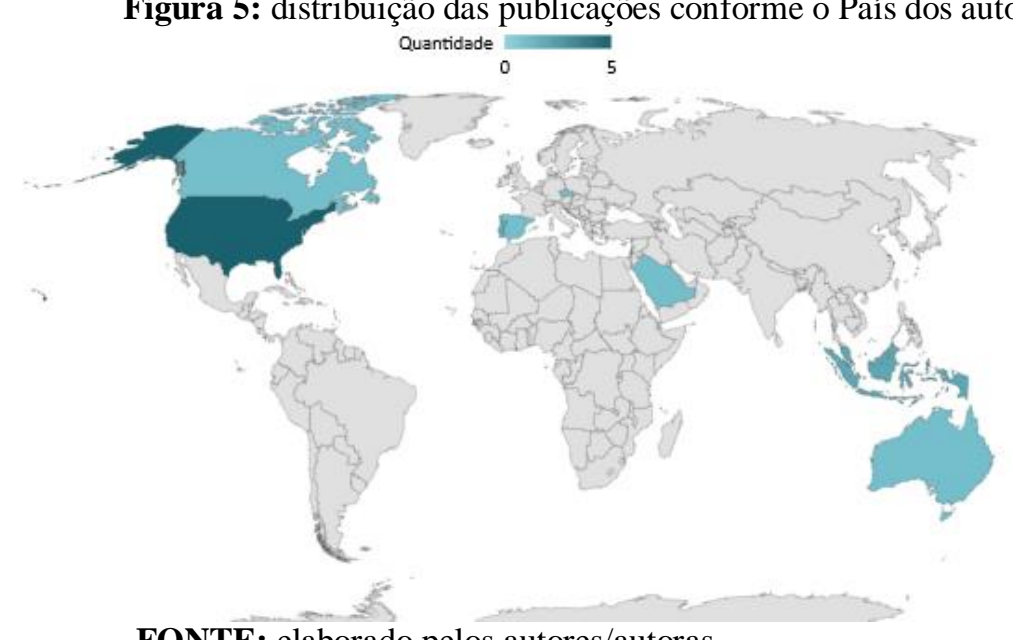

FONTE: elaborado pelos autores/autoras.

As instituições de ensino demandam o uso das tecnologias digitais e de plataformas para o aprendizado on-line, a fim de propiciar uma comunicação rápida, direta e colaborativa entre várias pessoas (JAMARI et al., 2014). A relação se torna independente das fronteiras geográficas, políticas ou econômicas (NINI, 2015). O estudante universitário, nesse contexto, usa suas mídias sociais constantemente, verificadas sempre de forma urgente (KORICH, 2016).

A urgência do estudante pode ser mitigada com a utilização pedagógica das mídias sociais. As mudanças constantes causadas pelo acesso imediato à informação, a forma que o conteúdo dos cursos é disponibilizado (cada vez mais inovador) devem continuar evoluindo e adaptando às novas maneiras que os estudantes aprendem (LIM; ANG; FUNG, 2017).

Os estudantes usam para se socializarem, nessa ordem, principalmente o Instagram, o Facebook, o Twitter e o YouTube. Nas preferências de utilização educacional, são eleitos o Facebook, o YouTube, o Instagram e o Twitter. Essas mídias são mais usadas até que os próprios e-mails dos alunos. Incorporá-las como ferramenta dentro da aprendizagem formal pode não aparecer com frequência (AL-BAHRANI; PATEL; SHERIDAN, 2015). No entanto, 


\section{REVISTAELETRÔNICADEGRADUACÄOO}

EPÓS-GRADUAÇÄO EM EDUCAÇÄO REJ/UFG

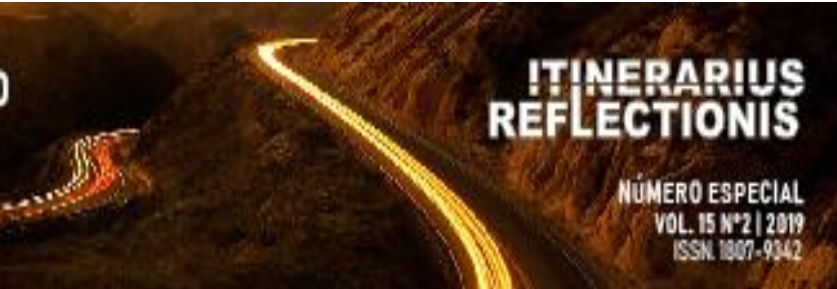

a abordagem de aprendizagem informal funciona de forma mais efetiva em processos colaborativos, como a criação de discussões e o desenvolvimento da escrita.

Adiante, os estudantes acreditam que as mídias sociais podem ser usadas como forma de aprendizado para o desenvolvimento de suas habilidades, a fim de gerar maior confiança nos seus estudos. Salienta-se que os alunos podem acessar conteúdos de cursos usando o Instagram com o foco em imagens (IRWANDANI; JUARIYAH, 2016).

O Instagram está presente em dispositivos móveis como smartphones e serve como um método de rápida disseminação de desafios educacionais que ratificam os conceitos dados em cursos. As imagens e os vídeos usados no feed do Instagram servem para facilitar a partilha do conteúdo em tempo real com os alunos. Ademais, as aulas gravadas em forma de vídeo podem incentivá-los a rever conteúdos, como uma forma de revisão e reflexão (KORICH, 2016).

Após o aluno realizar o upload do vídeo, é possível verificar o número de visualizações e os nomes das pessoas que gostaram dele. Esses dados podem ser rastreados, assim como o uso de hashtags vinculadas ao conteúdo. A contribuição do aluno sendo rastreada instiga o ensino dirigido e direcionado a um aprendizado mais eficiente e engajado (BLAIR; SERAFINI, 2014).

Apesar das restrições dos estudantes relacionadas à sua privacidade, ao medo de se expor no Instagram e ao receio de seus erros gramaticais, o Instagram oferece muitas vantagens, como mobilidade e acessibilidade por meio de smartphones, disseminação de conteúdo autêntico e oportunidades para interações significativas. $\mathrm{O}$ uso de imagens pela rede social explora a criatividade dos alunos por meio da construção de memes, de quadrinhos, entre outros produtos visuais eficazes, capazes de comunicar um conteúdo relevante aos propósitos educacionais (IRWANDANI; JUARIYAH, 2016).

Nesse ínterim, os memes podem ser usados como uma das estratégias criativas para o desenvolvimento de um processo de ensino e aprendizagem inovadores em línguas. A incorporação de imagens, texto escrito e tópicos especializados permite resultados bemsucedidos. Os alunos preferem este tipo de atividade com técnica simples, divertida, rápida e agradável a atividades dentro de sala de aula. A ideia, assim, da criação de memes é um método atraente, com frases cativantes (PURNAMA, 2017). 


\section{REVISTAELETRÔNICA DE GRADUAÇÄO}

EPÓS-GRADUAÇÄO EM EDUCAÇÄO REJ/UFG

REFITECTIONIS

NUMERO ESPECIAL

VoL. $15 K^{\circ} 212019$

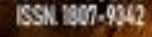

Quando descontraídos, os alunos não sentem que aprender línguas é uma obrigação. A competência comunicativa e intercultural dos alunos alcança estruturas e conteúdo linguísticos cada vez melhores nessas atividades de colaboração, consolidando o aprendizado. A maioria dos estudantes acredita que o intercâmbio cultural digital é importante para gerar uma aproximação do estilo de vida das pessoas de outras culturas e que ajuda na progressão da aprendizagem da língua. Além disso, a atividade na mídia digital serve para integrar quem estiver participando, ao reforçar laços entre os estudantes e os participantes. Portanto, facilita o contato com uma linguagem cotidiana, diminuindo distâncias físicas (PEDRÓ-GARCÍA, 2016).

As próprias funções de comunicação são melhoradas, além de ser um elemento motivador no processo de aprendizagem dos alunos. As atuais plataformas digitais podem servir como um encontro não apenas entre falantes nativos da língua, mas também entre grupos de alunos de diferentes origens, mas com objetivos similares. O potencial dessas aplicações no ensino de línguas é ilimitado e podemos encontrar na literatura experiências com e-mail, fóruns de discussão, bate-papos, sessões de videoconferência, entre outras. Salienta-se que não há dados suficientes sobre uma única plataforma que possa ser mais adequada às tarefas relacionadas com a aprendizagem de línguas (PEDRÓ-GARCÍA, 2016).

A mídia social é um dos produtos mais atuais e dinâmicos. É um método de ensino inovador com um impacto muito positivo na área de economia. A comunicação é facilitada na hora de fazer anúncios, posts sobre artigos econômicos relevantes ou vídeos. A comunicação direta é desenvolvida em contato direto com instrutores e permite que os alunos tímidos se comuniquem, façam conexões e melhorem sua experiência nessa aprendizagem. Além do mais, a colaboração entre os estudantes torna a aprendizagem uma experiência muito mais agradável. Os alunos matriculados em aulas em que a mídia social foi utilizada mostraram melhor engajamento. Portanto, há uma maior pontuação em testes de faculdade, demonstrando a eficácia desta nova ferramenta na economia (AL-BAHRANI; PATEL; SHERIDAN, 2015)(AL-BAHRANI; PATEL, 2015).

Espera-se que os professores adotem o maior número possível de produtos tecnológicos para promover a criatividade e motivação de seus alunos. Para obter um maior engajamento estudantil e diminuir o tédio dos alunos nas disciplinas, é importante que o 


\section{REVISTAELETRÔNICADEGRADUACÄO}

EPÓS-GRADUAÇÄOEM EDUCAÇÄO REJ/UFG

\section{ITINPARUS REFLECTIONIS}

NÜNERO ESPECIAL

VOL. $15 K^{+} 212019$

ISSN 100909

professor favoreça a autorregulação da aprendizagem. Ele pode introduzir oportunidades no uso de tecnologias digitais, para fomentar sua inserção no processo de aprendizagem real (PURNAMA, 2017)(YOT-DOMÍNGUEZ; MARCELO, 2017).

Esse processo de montar a atividade na mídia digital pode ser demorado para o instrutor, o que lhe traz muitas reservas. O preparo do material requer tempo e experiência com softwares diversos para a construção do conteúdo que resulte em mídia suportada pela plataforma de aprendizado eleita pelo instrutor. Esse tempo é uma carga de trabalho extra. Encontrar materiais úteis e preparar áudios e vídeos apropriados para o aplicativo demandam muito tempo de pesquisa (HAMAD, 2017).

Portanto, o ensino e a aprendizagem de hoje enfrentam mais desafios à medida que a tecnologia continua se atualizando. As redes sociais podem ter funções importantes no processo de aprendizado, mas ainda há muita pesquisa que precisa ser realizada, como a avaliação do conteúdo linguístico, a escolha de temas, a ortografia, a gramática entre os participantes, a avaliação curricular das atividades digitais, entre outras avaliações (PEDRÓGARCÍA, 2016) (AKHIAR et al., 2017).

\section{CONSIDERAÇÕES FINAIS}

A pesquisa ratificou a possibilidade de uso do Instagram para a promoção do aprendizado, por meio do planejamento educativo e com uma participação de toda comunidade acadêmica, professores, alunos, administração e setores da sociedade, mas não houve nenhuma proposta que tenha oferecido um modelo de sucesso real.

A intencionalidade pedagógica é um fator considerável para a utilização da rede social citada. Por meio dela, consideram-se os objetivos educacionais relevantes para que os estudantes alcancem suas competências demandadas pela sociedade contemporânea. Os autores dos trabalhos analisados procuram identificar ações pedagógicas que possam fazer um link entre a prática e a aplicação teórica com novas tecnologias, mas os estudos ainda percorrem por possibilidades estatísticas e investigações sobre os comportamentos de nossos alunos nas redes sociais. 


\section{REVISTAELETRÔNICADEGRADUACÄO}

EPÓS-GRADUAÇÄO EM EDUCAÇÄO REJ/UFG

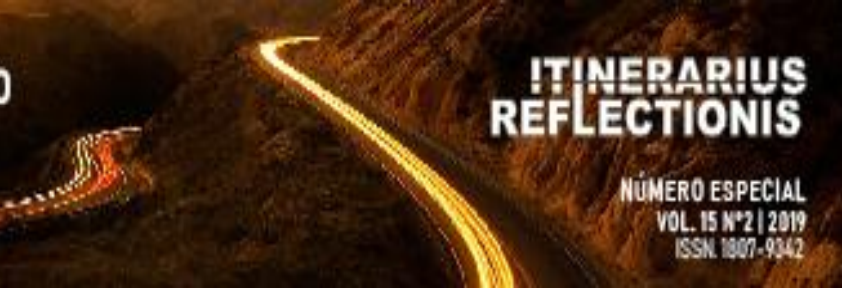

Nesse sentido, a metodologia Revisão Sistemática (RS) revelou amplitude relevante sobre as principais discussões em importantes bases de dados sobre o potencial do uso do Instagram no contexto educativo. Salienta-se que as práticas educativas com essa rede social ainda são um grande desafio, tendo em vista o surgimento recente do Instagram, bem como a sucinta articulação entre o planejamento educacional e a organização do trabalho pedagógico.

Ademais, observou-se que o Instagram possibilita estudos educativos para as variadas áreas do conhecimento, das humanas as exatas. Destacou-se o ensino de línguas e química permeadas pelas multimídias, com a premissa de textos curtos e reconhecimento de imagens. Por fim, elucidou-se que o mundo contemporâneo, de forma geral, utiliza as redes sociais para a articulação de variados conhecimentos, e despertar possibilidades de uso do Instagram no planejamento educativo formal é uma inquietação, por enquanto, nas práticas encontradas até o momento.

Temos que concordar que há uma dura e difícil jornada até encontrarmos soluções pedagógicas que sejam tecnológicas e ofereça um ambiente mediador, participativo e colaborador entre as práticas docentes e alunos conectados. Esta é a linha para futuras pesquisas.

\section{REFERÊNCIAS}

AKHIAR, Ayuni et al. Students' Perceptions and Attitudes Towards the Use of Instagram in English Language Writing. Malaysian Journal of Learning and Instruction, p. 47-72, 2017. Disponível em: 〈http://mjli.uum.edu.my/images/specialissue/2017/A3.pdf>.

AL-BAHRANI, Abdullah; PATEL, Darshak. Incorporating Twitter, Instagram, and Facebook in economics classrooms. Journal of Economic Education, v. 46, n. 1, p. 56-57, 2015.

AL-BAHRANI, Abdullah; PATEL, Darshak; SHERIDAN, Brandon. Engaging students using social media: The students' perspective. International Review of Economics Education, v. 19, p. 36-50, 2015. Disponível em: <http://dx.doi.org/10.1016/j.iree.2015.06.001>.

BARBOSA, Cláudia et al. Utilização do Instagram no ensino e aprendizagem de português língua estrangeira por alunos chineses na Universidade de Aveiro / The use of Instagram in the teaching and learning of Portuguese as a foreign language by Chinese students in the Un. of Aveir. Revista Latinoamericana de Tecnología Educativa - RELATEC, v. 16, n. 1, p. 21-33, 2017. Disponível em: <http://relatec.unex.es/article/view/2936>.

BLAIR, Risa; SERAFINI, Tina M. T.M. Integration of Education : Using Social Media Networks to Engage Students Social Media Statistics - 2014. IMSCI 2014 - 8th International Multi-Conference on Society, Cybernetics and Informatics, Proceedings, v. 12, n. 6, p. 


\section{REVISTAELETRÔNICADEGRADUACÄO}

EPÓS-GRADUAÇÄOEM EDUCAÇÄO REJ/UFG

28-31, 2014. Disponível em: <http://www.scopus.com/inward/record.url?eid=2-s2.084923265523\&partnerID=tZOtx3y1>.

CAMPBELL, Laurie O. Concept Mapping: An "Instagram" of Students' Thinking. The Social Studies, v. 107, n. 2, p. 74-80, 2016. Disponível em: <http://www.tandfonline.com/doi/full/10.1080/00377996.2015.1124377>.

CAPES, Coordenação de Aperfeiçoamento de Pessoal de Nível Superior. Documento de orientação de avalição: Critérios de Classificação Qualis - Ensino. Brasília. 2014. Disponível em: <https://www.capes.gov.br/images/stories/download/avaliacaotrienal/ Docs_de_area/qualis/ensino.pdf $>$.

CASTELLS, Manuel. A Sociedade em Rede. São Paulo: Paz e Terra, 1999. v. I. Disponível em:

<http://www.cies.iscte.pt/linhas/linha2/sociedade_rede/zip/Sociedade_em_Rede_CC.zip>.

COFFIELD, Frank et al. Learning styles and pedagogy in post-16 learning A systematic and critical review. Learning and Skills Research Centre, p. 84, 2004. Disponível em: <http://www.voced.edu.au/td/tnc_79.72>.

DE-LA-TORRE-UGARTE-GUANILO, Mônica Cecilia; TAKAHASHI, Renata Ferreira; BERTOLOZZI, Maria Rita. Revisão sistemática: noções gerais. Revista da Escola de Enfermagem da USP, v. 45, n. 5, p. 1260-1266, 2011.

DICHEVA, Darina. Gamification in Education: social network analysis. Proceedings of the 18th International Conference on Computer Systems and Technologies -

CompSysTech'17, June, p. 11-11, 2017. Disponível em: <http://dl.acm.org/citation.cfm?doid=3134302.3134305>.

FERREIRA-BORGES, Flavio; FRANÇA-TELES, Lucio. El uso de redes sociales en la práctica educativa de una asignatura de postgrado: una investigación sobre el uso de las TRIC. index.comunicación, v. 9, n. 1, p. 109-125, apr. 2019. ISSN 2174-1859. Disponível em: <http://journals.sfu.ca/indexcomunicacion/index.php/indexcomunicacion/article/view/444>.

GALVÃO, Taís Freire; PANSANI, Thais de Souza Andrade; HARRAD, David. Principais itens para relatar Revisões sistemáticas e Meta-análises: A recomendação PRISMA.

Epidemiologia e Serviços de Saúde, v. 24, n. 2, p. 335-342, 2015. Disponível em: <http://www.iec.pa.gov.br/template_doi_ess.php?doi=10.5123/S167949742015000200017\&scielo=S2237-96222015000200335>.

HAMAD, Mona M. Using WhatsApp to Enhance Students' Learning of English Language "Experience to Share". Higher Education Studies, v. 7, n. 4, p. 74, 2017. Disponível em: <http://www.ccsenet.org/journal/index.php/hes/article/view/71758>.

IRWANDANI, Irwandani; JUARIYAH, Siti. Pengembangan Media Pembelajaran Berupa Komik Fisika Berbantuan Sosial Media Instagram sebagai Alternatif Pembelajaran. Jurnal Ilmiah Pendidikan Fisika Al-Biruni, v. 5, n. 1, p. 33, 2016. Disponível em: <http://ejournal.radenintan.ac.id/index.php/al-biruni/article/view/103>.

JAMARI, Diyana et al. Learning Through Social Media : Students' Perception. Man In 
India, v. 97, n. 19, p. 263-273, 2014.

KENSKI, Moreira Vani. Educação e tecnologias: o novo ritmo da informação. São Paulo: Papirus, 2008a.

KENSKI, Vani Moreira. Novos processos de interação e comunicação no ensino mediado pelas tecnologias. Cadernos de Pedagogia Universitária, p. 24, 2008b. Disponível em: <http://www.prpg.usp.br/attachments/article/640/Caderno_7_PAE.pdf>.

KORICH, Andrew L. Harnessing a Mobile Social Media App to Reinforce Course Content. Journal of Chemical Education, v. 93, n. 6, p. 1134-1136, 2016.

LÉVY, Pierre. As Tecnologias da Inteligência: O Futuro do Pensamento na Era da Informática. Rio de Janeiro (RJ), Editora 34, 1996.

XUTAO, Li; TUAN-ANH, Nguyen Pham; GAO, Cong; QUAN, Yuan; XIAO-LI, Li; SHONALI, Krishnaswamy. Where you Instagram?: Associating Your Instagram Photos with Points of Interest. In Proceedings of the 24th ACM International on Conference on Information and Knowledge Management(CIKM '15). ACM, New York, NY, USA, 12311240. 2015. DOI: https://doi.org/10.1145/2806416.2806463.

LIM, Rachel Rui Xia; ANG, Alina Sihui; FUNG, Fun Man. Application of Social Media in Chemistry Education: Incorporating Instagram and Snapchat in Laboratory Teaching.

Teaching and the Internet: The Application of Web Apps, Networking, and Online Tech for Chemistry Education. Washington, DC: American Chemical Society, 2017. p. 37-53. Disponível em: <http://pubs.acs.org/doi/abs/10.1021/bk-2017-1270.ch003>.

MCKNIGHT, K.; O’MALLEY, K.; RUZIC, R.; HORSLEY, M. K.; FRANEY, J. J.y BASSETT, K. Teaching in a Digital Age: How Educators Use Technology to Improve Student Learning. Journal of Research on Technology in Education, 48(3), 194-211. 2016.

MORAN, J. M. Ensino e aprendizagem inovadores com tecnologias audiovisuais e telemáticas. En J. M. MOrAn; M. T. MAsettO y M. A. Behrens (Orgs.), Novas Tecnologias e Mediação Pedagógica (10a). Campinas/SP: Papirus. 2006.

MOREIRA, José António; MONTEIRO, Angélica Maria Reis. Formação e ferramentas colaborativas para a docência na web social. Revista Diálogo Educacional, v. 15, n. 613, p. 379, 2015. Disponível em:

<http://www2.pucpr.br/reol/index.php/dialogo?dd1=15314\&dd99=view>.

NINI, Eddy. Private Open Source Social Networking Media for Education. 2015 Fifth International Conference on e-Learning (econf), p. 220-224, 2015. Disponível em: <http://ieeexplore.ieee.org/lpdocs/epic03/wrapper.htm?arnumber=7478235>.

PAGÁN, Liz M.; ORTIZ, Laurie A. Web social y alfabetización informacional : experiencia en la Universidad de Puerto Rico Web social y alfabetización informacional : experiencia en la Universidad de Puerto Rico. e-Ciencias de la Información, v. 7, n. 1, 2017.

PEDRÓ-GARCÍA, Andreu. Instagram: una experiència de telecol·laboració australianocatalana. REIRE Revista d'Innovació i Recerca en Educació, v. 9, n. 1, p. 1128, 2016. Disponível em: <http://revistes.ub.edu/index.php/REIRE/article/view/14629>. 


\section{REVISTAELETRÔNICADE GRADUAÇÄO}

EPÓS-GRADUAÇÄO EM EDUCAÇÄO REJ/UFG

\section{ITINERARIS REFLECTIONIS}

NÚMERO ESPECIAL

VOL. $15 \mathrm{~K}^{\top} 2 \mid 2019$

PURNAMA, Agnes Dian. Incorporating Memes and Instagram to Enhance Student's

Participation. LLT Journal: A Journal on Language and Language Teaching, [S.1.], v. 20, n. 1, p. 1-14, mar. 2017. ISSN 2579-9533. Disponível em: <http://ejournal.usd.ac.id/index.php/LLT/article/view/404>.

SANTOS, Valmaria Lemos da Costa; SANTOS, José Erimar Dos. As Redes Sociais Digitais e sua Influência na Sociedade e Educação Contemporâneas. HOLOS, v. 6, p. 307, 2015. Disponível em: <http://www2.ifrn.edu.br/ojs/index.php/HOLOS/article/view/1936>.

SMITH, Erika E. Social media in undergraduate learning: categories and characteristics. International Journal of Educational Technology in Higher Education, v. 14, n. 1, 2017.

SONG, Yanjie. Methodological Issues in Mobile Computer-Supported Collaborative Learning (mCSCL): What Methods, What to Measure and When to Measure ? Educational Technology \& Society, v. 17, n. 4, p. 33-48, 2014. Disponível em:

<http://www.jstor.org/stable/jeductechsoci.17.4.33>

STATISTA. Leading global social networks 2018 | Statistic. The Statistics Portal.

Disponível em: <https://www.statista.com/statistics/272014/global-social-networks-rankedby-number-of-users/>. , 2018

UNESCO. Diretrizes de políticas da UNESCO para a aprendizagem móvel. Organização das Nações Unidas para a Educação, a Ciência e a Cultura (UNESCO), 2014. Disponível em: <http://unesdoc.unesco.org/images/0022/002277/227770por.pdf>.

YOT-DOMÍNGUEZ, Carmen; MARCELO, Carlos. University students' self-regulated learning using digital technologies. International Journal of Educational Technology in Higher Education, v. 14, n. 1, 2017. 